\title{
Problematización del acoso escolar en estudiantes: una alternativa de intervención
}

\section{Problematization of school bullying in students: an alternative intervention}

Problematização do bullying em estudantes: uma alternativa de intervenção

María de Guadalupe Jocobi Zúñiga

Instituto Tecnológico de Sonora, Sonora, México jocobi94@hotmail.com https://orcid.org/0000-0002-1126-9369

Oscar Cruz Pérez

Universidad de Ciencias y Artes de Chiapas, Chiapas, México oscar.cruz@unicach.mx https://orcid.org/0000-0003-2452-2834

Jesús Ocaña Zúñiga Universidad de Ciencias y Artes de Chiapas, Chiapas, México jesus.ocana@unicach.mx https://orcid.org/0000-0003-0300-0797

Germán Alejandro García Lara

Universidad de Ciencias y Artes de Chiapas, Chiapas, México german.garcia@unicach.mx https://orcid.org/0000-0002-4075-4988 
Revista Iberoamericana

de las Ciencias Sociales y

Humanísticas

ISSN: $2395-7972$

\section{Resumen}

En este artículo se presentan los resultados de una investigación que se enfocó en una estrategia de intervención para discutir el tema del acoso y la violencia en el contexto escolar. Se trata de una investigación cualitativa que incluyó grupos de discusión, así como la observación participante de 33 estudiantes de una secundaria pública de la localidad de Ciudad Obregón, Sonora, en el municipio de Cajeme. El análisis se enfocó en la manera de convivir de los jóvenes y la problematización del acoso escolar por parte de los adolescentes. Los resultados sugieren que los alumnos son capaces de identificar el acoso escolar como un problema que está presente en la convivencia con sus pares, basado en agresiones tanto físicas como verbales que evidencian un abuso de poder, intencionalidad y repetición entre los involucrados. Sus percepciones sobre la problemática demuestran que existe una convivencia hostil entre los agresores, irrespeto entre los compañeros y problemas de comunicación.

Palabras clave: adolescentes, acoso escolar, normalización de la violencia.

\section{Abstract}

The article presents the results of a research focused on an intervention strategy where bullying and violence in the school context are discussed. This is a qualitative research that included discussion groups and participant observation of 33 students from a public secondary school in the city of Ciudad Obregón, Sonora, in the municipality of Cajeme. The analysis focuses on the way young people coexist and the problematization of school bullying by teenagers. The results suggest that teenagers are capable of identifying bullying as a problem that is present in coexistence with their peers, based on both physical and verbal aggressions, where abuse of power, intentionality and repetition exist among those involved. The perception of the problem

refers to the bad coexistence that occurs between the aggressors, it is mentioned that there is no respect among their peers, as well as communication problems.

Keywords: bullying, teenagers, normalization of violence, violence. 
Revista Iberoamericana

de las Ciencias Sociales y

Humanísticas

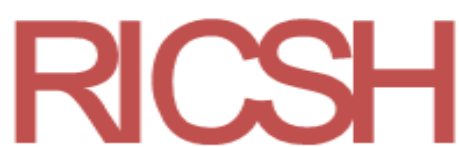

ISSN: $2395-7972$

\section{Resumo}

Este artigo apresenta os resultados de uma investigação focada em uma estratégia de intervenção para discutir a questão do bullying e da violência no contexto escolar. Trata-se de uma investigação qualitativa que incluiu grupos de discussão, bem como a observação participante de 33 alunos de uma escola pública na cidade de Ciudad Obregón, Sonora, no município de Cajeme. A análise enfocou o modo de vida dos jovens e a problematização do bullying por parte dos adolescentes. Os resultados sugerem que os alunos são capazes de identificar o bullying como um problema presente na convivência com os colegas, com base em agressões físicas e verbais que mostram abuso de poder, intencionalidade e repetição entre os envolvidos. Suas percepções sobre o problema mostram que existe uma convivência hostil entre os agressores, desrespeito entre os parceiros e problemas de comunicação.

Palavras-chave: adolescentes, bullying, normalização da violência.

Fecha Recepción: Octubre 2018

Fecha Aceptación: Junio 2019

\section{Introducción}

En este artículo se analizan las manifestaciones de acoso y sus significaciones en estudiantes de secundaria de una localidad del municipio de Cajeme, Ciudad Obregón, Sonora. Esta ciudad es actualmente una de las más conflictivas y peligrosas en el estado de Sonora debido al crimen organizado, los actos de violencia y el vandalismo, lo cual tiene efectos negativos en los comportamientos y actitudes de los jóvenes (Gómez y Gómez- Maqueo, 2013).

Para Orue y Calvete (2012), el ser testigo de la violencia familiar, ver contenidos de esa índole por algún medio de comunicación o ser víctima actos semejantes en sus diversas modalidades puede propiciar la agresividad y la aceptación de la violencia. En otras palabras, se llega a una normalización de esas conductas por la interacción reiterada con ellas (Hernández, 2015).

Según datos de la Encuesta Nacional de Victimización y Percepción sobre Seguridad Pública (Envipe), en el año 2012, en México, $32.4 \%$ de los hogares fueron víctimas de algún delito, porcentaje que se incrementó en 2016 a 34.2\%. Durante el año 2016 se cometieron 31.1 millones de delitos, de los cuales 24.2 millones se asocian a robos o asaltos, extorción, fraude, amenazas verbales, lesiones, entre otros. De hecho, a nivel nacional, $61.1 \%$ de la población de 


\section{Revista Iberoamericana \\ de las Ciencias Sociales y \\ Humanísticas}

ISSN: $2395-7972$

más de 18 años considera que la inseguridad y la delincuencia son los problemas más importantes en cuanto a seguridad pública (Instituto Nacional de Estadística y Geografía [Inegi], 2017).

Este tipo de acciones, lógicamente, también se pueden encontrar en la escuela, pues los episodios de agresión entre compañeros (bullying) son cada vez más frecuentes. En este sentido, los resultados del Programa para la Evaluación Internacional de Alumnos (PISA) en México muestran que los estudiantes de sexo masculino son más propensos que las mujeres a ser victimarios de acoso escolar en las instituciones. Este es un fenómeno que se puede definir como aquella conducta agresiva que se caracteriza no solo por su intencionalidad, repetición y diferencias de poder, sino también por generarse con la participación de tres actores principales: víctima, observador y agresor (Olweus y Limber, 2017).

Estas agresiones pueden implicar maltratos físicos o verbales que se realizan de forma directa hacia la víctima (Ferrel, Cuan, Londoño y Ferrel Ballestas, 2015) o también de modo indirecto causando daños a la propiedad privada (Enríquez y Garzón, 2015). Según diversos estudios, se ha podido establecer que las conductas violentas del ser humano pueden ser influenciadas por diversos factores, entre los que destacan los ambientales, los familiares, los sociales y los personales (Carrasco, García y Zaldívar, 2018). En el caso de los factores de riesgo del acoso escolar, se ha encontrado evidencia de que la exposición a la violencia contribuye a la agresividad de los individuos (Carrasco, García, y Zaldívar, 2018; Orue y Calvete, 2012), así como los estilos de crianza, pues crecer en un clima familiar negativo fomenta las conductas agresoras de los individuos (Valdés y Carlos, 2014; Valdés, Carlos y Torres, 2012).

Los adolescentes que asumen estas conductas suelen tener menor desempeño académico y más problemas de disciplina en la escuela, a diferencia de quienes no actúan de esa manera, los cuales tienen un mejor autoconcepto prosocial (Valdés, Bautista, Vera y Herrera, 2013). Asimismo, los agresores suelen presentar problemas de trastornos psicológicos como impulsividad, baja tolerancia a la frustración, conductas antisociales y problemas escolares (Albores, Sauceda, Ruiz y Roque, 2011). Las víctimas, en cambio, refieren daños hacia su persona, ideación e intento de suicidio, fobia escolar, temor irracional y depresión (Arroyave, 2012).

Para atender este complejo fenómeno se han desarrollado intervenciones dirigidas a estudiantes de nivel básico, las cuales han ofrecido resultados favorables en cuanto a las conductas agresoras y el bienestar del alumnado. De hecho, en intervenciones reportadas en distintos países se ha trabajado con variables que no son estrictamente psicológicas, como la 


\section{Revista Iberoamericana \\ de las Ciencias Sociales y Humanísticas}

ISSN: $2395-7972$

educación para la paz, los derechos humanos, la convivencia y los valores prosociales (Garaigordobil, 2010; Garagordobil y Maganto, 2011). Otros programas sobre acoso escolar se han enfocado en el autodominio, la expresión y la comprensión de los propios sentimientos, la asertividad, la empatía, el autoestima, la resolución de problemas para el control de la ira y la resolución de conflictos personales (Caballo, Caldero, Carrillo, Salazar e Irurtia, 2011; Cerezo y Sánchez, 2013; Ferreira y Reyes, 2011; Güemes, 2011; Wong, Cheng, Ngan y Stephen, 2011),

En el caso de México, existen programas de intervención diseñados para la disminución de conductas agresoras en las escuelas. Por ejemplo, la Secretaría de Educación Pública (SEP) ha diseñado el Programa Nacional de Convivencia Escolar (PNCE) con la finalidad de impulsar la autoestima, el manejo de las emociones, las normas de resolución de conflictos y la participación de las familias (Fierro, 2016). Aunado a esto, sobresalen los estudios cualitativos que se han realizado en los centros educativos, los cuales han aportado información que sirve para entender las distintas aristas de la violencia escolar (Mayora y Castillo, 2014). En este sentido, Paulín (2015) encontró en los relatos de sus participantes aspectos vinculados con la normalización y justificación de la violencia; esto se evidencia en sus concepciones sobre las peleas como única vía para resolver los conflictos. Estos resultados, por supuesto, resultan alarmantes, de ahí el interés por estudiar el acoso escolar desde la percepción de los alumnos, pues de ese modo se pueden proponer e implementar estrategias para atender dicho problema (Orue y Calvete, 2012).

Por eso, el objetivo del presente trabajo fue comprender las manifestaciones del acoso escolar y sus significaciones en estudiantes de nivel secundario de una localidad ubicada en el noroeste de México, donde los actos de violencia y el vandalismo se hallan muy latentes.

\section{Proceso metodológico}

La investigación se realizó desde una perspectiva cualitativa, posicionada en un paradigma sociocrítico para analizar las transformaciones sociales y la solución de problemas teniendo como punto de partida la autorreflexión (Alvarado y García, 2008). Con este tipo de estudios se intenta una descripción del mundo natural desde la perspectiva de los individuos, para lo cual se analizan sus experiencias individuales o grupales, sus interacciones y sus comunicaciones mientras las producen (Gibbs, 2012). 
Revista Iberoamericana

de las Ciencias Sociales y

Humanísticas

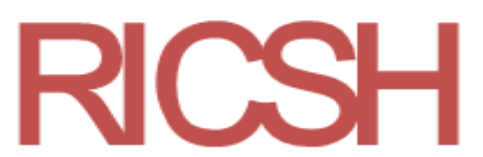

ISSN: $2395-7972$

\section{Participantes}

Los participantes en esta investigación fueron 33 adolescentes (60\% de sexo femenino y $40 \%$ de sexo masculino) que estudiaban segundo grado en una escuela secundaria ubicada en Ciudad Obregón, municipio de Cajeme, Sonora. Las edades de los participantes oscilaban entre los 13 y los 14 años. Los criterios de inclusión fueron que los alumnos tuvieran antecedentes de conductas agresoras.

\section{Técnicas de recolección de datos}

La recolección de información se realizó a través de un grupo de análisis para problematizar sobre el acoso escolar, sus actores y consecuencias. El análisis para la problematización es un procedimiento de producción de información (discursos) a través de la reflexión colectiva de un fenómeno, condición o situación que exige ser cuestionado, repensado e interrogado, lo cual permite construir nuevos discursos y prácticas individuales y colectivas para superarlos. Para ello, es importante la coordinación de un moderador que al mismo tiempo participa, observa y recoge información valiosa para dar cuenta del proceso y de los productos que genera el grupo.

\section{Procedimiento}

Se solicitó autorización en la institución educativa para realizar la intervención con el grupo; luego se seleccionó a los estudiantes que estarían en el programa, los cuales participaron de forma voluntaria. Después se aplicó una intervención estructurada en 10 sesiones de 50 minutos cada una, las cuales sirvieron para recoger sus discursos. Es importante mencionar que la recolección de la información empírica resultó muy dificultosa por el número de participantes, de ahí que no se pudieran documentar todos los discursos que circularon en el grupo. Aun así, los datos recabados permiten dibujar la realidad que comparten los alumnos en la escuela.

Para examinar los datos se siguió el siguiente procedimiento: el contenido del grupo de discusión se transcribió en Word y luego fue analizado según el enfoque inductivo propuesto por Thomas (2003). De este modo surgieron los temas más representativos o significativos, a los cuales se les estableció una relación clara entre las categorías o tópicos. En concreto, el análisis de la información se realizó mediante el software Atlas ti (versión 7). 


\section{Resultados}

En este trabajo se buscó problematizar con los adolescentes el tema del acoso escolar presente en su escuela; para ello, se trataron las siguientes temáticas:

1. Mi experiencia en la escuela.

2. ¿Qué es el acoso escolar?

3. El significado de la escuela.

4. El acoso escolar en su escuela.

5. Actores en el acoso escolar.

6. Los tipos de acoso escolar.

7. Las consecuencias del acoso escolar.

8. Las consecuencias de los actores en el acoso escolar.

A partir de la información empírica recolectada se construyeron tres categorías: a) contexto de los estudiantes en la escuela, $b$ ) dinámica de los adolescentes y $c$ ) problematización del acoso escolar.

\section{El contexto de los estudiantes en la escuela}

Definida como aquellos comportamientos y actitudes que mejoran las relaciones interpersonales de los estudiantes (Fernández-Batanero, 2015), la convivencia escolar es uno de los elementos más importantes para propiciar la interacción de los adolescentes. En el caso concreto de los participantes de esta investigación, la manera de socializar se basa en juegos bruscos. Los integrantes, de hecho, comentan que su convivencia se fundamenta en la violencia, lo cual incluye golpes, groserías, críticas, burlas basadas en las características físicas, exclusión social y acoso en redes sociales.

Los hombres de mi salón son un tanto violentos entre sí (1:57).

Nos llevamos un poco a golpes yo y mis amigos de otros salones (1:23).

Nos burlamos de los demás por ser chistosos o por otra cosa, pero lo malo es que tienes que venir con tenis de color negro, blanco y azul y eso no me gusta a mí y a las demás personas (1:52).

Es como que muy mal porque algunos de mis compañeros agreden a un compañero que va con nosotros; le dicen cosas, le pegan y así hasta que lo hacen llorar: son muy peleoneros (1:29). 
Revista Iberoamericana

de las Ciencias Sociales y

Humanísticas

ISSN: $2395-7972$

Me quería pegar y cosas así en los recesos, me decía que estaba muy pendeja, cosas así, me ponía indirectas por Facebook (1:71).

En estos discursos se evidencia que la manera de interactuar entre pares es mediante conductas violentas, las cuales son habituales en el contexto escolar. Las agresiones, asimismo, son una de las principales maneras para socializar debido a que pareciera que no conocen otra manera de interactuar con los compañeros. En pocas palabras, estas situaciones son concebidas como normales. "Pues es que hay pleitos, pero son inofensivos” (23:1); "es juego, ya que... ¡sin bullying no hay amistad!" (2:93). Estos comentarios son una clara evidencia de que los adolescentes conviven con la violencia, la cual no suele ser percibida como un aspecto negativo (Paulín, 2015).

Sin embargo, vale acotar que para algunos jóvenes este ambiente hace que se sientan incómodos y con deseos de huir de él (Barquero, 2014):

A veces me dan ganas de cambiarme de escuela porque muchos se burlan de

lo que haces. Con mis compañeras que me junto, muchas veces andan insoportables, se burlan de lo que dice, que si porque lo dijiste mal o algo así (1:71).

No puedes disfrutar ni poner atención porque tus compañeros te molestan, donde hay maestros injustos y malas amistades (3:26).

Como se aprecia, el ambiente en esta institución está generando que los estudiantes no se sientan cómodos en su grupo, lo cual puede ocasionar distintos problemas, entre los que se pueden destacar el ausentismo, la deserción escolar, así como los problemas académicos y de conducta (Arroyave, 2012).

Estos hallazgos contradicen el ideal fijado por cualquier escuela, espacio donde los estudiantes deberían desarrollarse de forma social y académica, y donde se deberían promover comportamientos pacíficos, así como conductas y actitudes positivas que influyan en el buen clima escolar, en el rendimiento académico y en las relaciones interpersonales (Córdoba, Del Rey, Casas y Ortega, 2016; Ossa, Figueroa y Rodríguez, 2016; Viguer y Solé, 2015). En el grupo de participantes, sin embargo, se percibe un ambiente de socialización negativo que se materializa en agresiones físicas y verbales que buscan causar daños a los pares (Barquero, 2014). 
Revista Iberoamericana

de las Ciencias Sociales y

Humanísticas

ISSN: $2395-7972$

\section{Dinámica de los adolescentes}

El escenario de los adolescentes es planteado como con conjunto de conflictos en la convivencia. En concreto, el acoso físico es vinculado con juegos bruscos, empujones, patadas, peleas y golpes cotidianos.

Pues a veces se insultan, se dicen cosas, pero al rato ya andamos bien; dicen sobrenombres y, pues, se portan mal (4:87).

Muy bruscos algun@s, pero otros bien; son muy buenos con sus amigos, pero otros son muy malos: insultan, se burlan, agreden y no les importa (4:63).

Se golpean entre sí, se agreden, se insultan entre sí, etc. (4:38).

Bien, a veces un poco mal y nos golpeamos un poco (4:25).

En estos relatos se puede observar que ellos mismos no se identifican como agresores o como participantes del fenómeno, sino que se alejan del problema verbalizándolo de forma impersonal: "Se golpean, se gritan, se pegan patadas" (4:50). Esto demuestra cierta indiferencia hacia ese tipo de acciones. También hacen referencia a las agresiones verbales que surgen con apodos, groserías, insultos, burlas, gritos y "carrilla" como parte de la convivencia entre sus compañeros de clase.

Pues a veces se insultan, se dicen cosas, pero al rato ya andamos bien, dicen sobrenombres y pues se portan mal (4:87). Pues se comportan algunas veces bruscamente y se burlan unos de otros, pero así es como convivimos (4:68). Se golpean, se gritan, se pegan patadas (4:50).

Estas conductas las realizan grupos conflictivos que hacen que las víctimas se excluyan de los demás y no puedan comunicar las agresiones que reciben: "Más o menos, no se hablan. No conviven muy bien. Ni se hablan" (4:77).

Según se puede inferir de estos discursos, existe una "normalización de la violencia", pues las agresiones son obviadas, las niegan e incluso participan en actos violentos: "Hablando y jugando con ellos, corriendo, etc. Nos llevamos brusco, pero bien" (4:18). En otras palabras, sus dinámicas se basan en agresiones, aunque no son capaces de identificarlas como conductas negativas. 
Revista Iberoamericana

de las Ciencias Sociales y

Humanísticas

ISSN: $2395-7972$

A lo que yo sé bien, se llevan bien, aunque a veces puede haber conflictos, pero luego se arregla (4:99).

Bien, nos llevamos bien, alguno que otro problema, pero bien, a veces pesado, pero lo arreglamos, nos ponemos apodos de cariño y nos llevamos bien $(4: 61)$.

Muy bruscos algun@s, pero otros bien, son muy buenos con sus amigos (4:62).

Dentro de la contextualización de la problemática en la escuela, los participantes hablan sobre una convivencia negativa y hacen referencia a las agresiones con las cuales conviven los integrantes del grupo, pues mencionan que ellos realizaban agresiones físicas y verbales. Estos hallazgos concuerdan con investigaciones que explican que las conductas violentas en los centros educativos forman parte de la interacción de los jóvenes (Maschio et al., 2015; Vega y González, 2016).

\section{Problematización del acoso escolar}

En este trabajó se buscó problematizar con los adolescentes el tema del acoso escolar y los actos de violencia. Con esta estrategia se cuestiona, se reflexiona y se interroga a los participantes sobre un tema que ellos conciben como normal. Específicamente, y a través de preguntas abiertas, se cuestionaron sus saberes sobre el acoso escolar, las conductas y las actitudes de acoso, así como sus participaciones y las consecuencias que ello genera. Al mismo tiempo, se interrogó sobre las afectaciones que esas acciones tienen para las víctimas.

En los relatos, los participantes mencionaron que los distintos actores del acoso escolar comparten características; por ejemplo, el agresor y observador tienen en común el vigilar a las víctimas y la participación en las agresiones tanto físicas como psicológicas. Por otro lado, la victima comparte con el observador el miedo a ser agredido y el silencio sobre la problemática. Los alumnos hablaron del agresor como un individuo que abusa de su poder, lo cual materializa con empujones, insultos, golpes, burlas y patadas.

Le pega y abusa o le dice cosas (5:1), agrede a las personas física y psicológicamente (5:8), agrede a los demás (5:25), hiere a la víctima, la lastima (5:71), es el que le pega a la víctima, le agrede física y psicológicamente (5:74). Quien le pega a alguien más por nada, porque el agresor a lo mejor recibe ese mismo trato que le da a sus compañeros (5:92). 
Revista Iberoamericana

de las Ciencias Sociales y

Humanísticas

ISSN: $2395-7972$

Los alumnos mencionaron que esta persona es quien vigila a la víctima y es capaz de torturarlo y amenzarlo:

Sigue a la persona a la cual es seguida y la golpea para ocacionarle daño (5:38).

Tortura a la víctima física y moralmente con insultos o golpes (5:89). Es al que le pegan y que lo tienen amenazado para que no le diga a los maestros o a sus papás (5:93).

Para los adolescentes un agresor es un individuo que provoca peleas con el objetivo de desahogarse, manifestar odio o conseguir poder:

Es el que agrede a equis persona por gusto o para desahogarse de sus problemas personales (5:59). Provoca peleas física y verbalmente (5:21). Agrede a los demás, a las que son débiles (5:29).

Estos discursos dan cuenta de una convivencia llena de juegos bruscos, peleas y bromas pesadas. Los participantes reconocieron que muchos de sus compañeros actúan o han actuado como agresores de otros alumnos.

En lo que se refiere a la identificación de la víctima, los adolescentes la identifican como un individuo que recibe las agresiones fisicas y verbales y que es vigilado por su agresor o agresores. Este actor, según los participantes, es humillado y suele tener miedo, lo que provoca que sea incapaz de defenderse. Otra característica que destacan es el silencio, pues las víctimas no hablan del problema.

Se queda callado y lo lastiman (5:48).

Al que agreden, pues muchas veces se queda callado por miedo y otras veces lo acusa (5:101).

Es golpeado, agredido, insultado, pateado, burlado, empujado (5:66).

Es al que siguen y agreden en la escuela o así (5:39).

No dice nada por miedo y es el que sufre y al que golpean (5:87).

En lo que se refiere al observador, los participantes lo describieron como un individuo que contempla el suceso de acoso y no es capaz de actuar ante el acontecimiento:

Mira cómo le pegan y lo agreden (5:3). Al que ve sin decir nada (5:10). Es el que mira, pero no dice nada y, pues, nomás mira y no hace nada (5:88). 
Revista Iberoamericana

de las Ciencias Sociales y

Humanísticas

ISSN: $2395-7972$

Los estudiantes se expresan del observador como alguien que no apoya a sus compañeros agredidos debido a que no se quiere convertir en otra víctima:

Ve lo que está pasando, pero no dice nada, por miedo a convertirse en victima (5:43). Es el que nomás ve la agresión y no dice nada por miedo a que le hagan lo mismo (5:46). Mira y no dice nada por temor a que le hagan daño (5:49). El observador mira como le pegan a la víctima y no va y dice, porque tiene miedo que le pase lo mismo, que el agresor le pegue (5:54).

Para los participantes los tipos de acoso escolar son el psicológico, el físico y el ciberacoso. Según sus discursos, el acoso psicológico es aquel que no implica golpes, sino que tiene que ver con humillar, desvalorizar y excluir a los compañeros. También mencionaron que en el acoso escolar se agrede de forma verbal por medio de críticas, apodos, groserías, burlas, insultos y ofensas.

Es cuando te dicen cosas, pero no te golpean (6:1). Es cuando agreden u ofenden con palabras (6:4). Le dice cosas hirientes al niño ahí en la escuela (6:21). Cuando hacen sentir menos a una persona y él se siente mal (6:29). Te alejas de todo, te rehúyen, te quedas solo o sola (6:56). Cuando te dicen groserías o le dicen algo ofensivo con palabras y que no se respeta (6:69).

En cuanto al acoso físico, hacen referencia al contacto directo entre los involucrados; este se da en la modalidad de peleas, empujones, arañazos, jalones, golpes, puñetazos y patadas:

Es cuando la agresión se da mediante el contacto físico ya sea por medio de golpes, empujones, etc. (6:35). Es cuando golpean sin razón, agreden golpeando en el cuerpo (6:39). Es el que se reacciona con golpes físicos con patadas y puñetazos para agredir (6:68). Es aquella que es con golpes, empujones, puños, arañazos, etc. (6:64).

En relación con el ciberacoso, sostuvieron que las agresiones se dan mediante las redes sociales, enviando fotos o haciendo memes a las víctimas para ridiculizarlas, exhibirlas o denostarlas:

Se le ofende por medio de las redes sociales (6:23). El que ofende por las redes sociales (6:30). Cuando le dicen muchas cosas por medio de las redes sociales (6:78). Es cuando hacen memes o fotos solo para que te molesten porque es vergonzoso (6:7). 


\section{Revista Iberoamericana \\ de las Ciencias Sociales y Humanísticas}

ISSN: $2395-7972$

Los estudiantes reconocieron que tanto ser víctima como victimario de acoso o violencia en la escuela tiene fuertes consecuencias. En el caso del victimario, tiene problemas sociales (con sus compañeros como grupo) y también de manera individual (con sus amigos y con las autoridades de la escuela). En cuanto a los problemas escolares, los participantes mencionaron que los agresores suelen tener reportes por sus conductas hacia los pares:

Tener muchos problemas en la escuela y con sus familiares (7:18). Que los reporten los maestros por no hacer caso (7:45).

En lo concerniente a las víctimas, es muy frecuente ver en ellos heridas en el cuerpo (p. ej., cicatrices, moretes, arañones, mordeduras y cortadas). Además, profundizaron en las consecuencias de orden psíquico, sobre las posibilidades de generar "traumas" como la depresión y los deseos de esconderse de los demás o volverse callados. Sostuvieron que las heridas del cuerpo pueden sanar, pero los traumas psicológicos son difíciles de resolver.

Con el trabajo de reflexión de las diferentes temáticas se logró que los adolescentes identificaran que los comportamientos de acoso y de violencia que aparecían como normales y naturales en la vida cotidiana de la escuela se percibieran como acciones problemáticas que dañan a todos:

El acoso escolar es algo muy feo, pero por desgracia es normal (9:73).

Sé que el acoso escolar es algo muy malo, porque podemos dañar a las personas $(9: 30)$.

Lo que yo considero es que no está nada bien, que lo niños agreden a otros compañeros, pero esos problemas bien sabemos que vienen de casa (9:86). Pues es malo, no lo debo de hacer ni permitir que me lo hagan y, pues, no es justo que lo hagan (9:92).

El acosador en la víctima provoca varios traumas, pero los puede evitar avisando a las autoridades para que acabe el acoso y no sigan molestando $(9: 21)$.

Los adolescentes identifican el acoso escolar como un problema que está presente en la convivencia entre sus compañeros, y que este se manifiesta en agresiones tanto físicas como verbales, con abuso de poder, intencionalidad y de forma reiterativa: 
Revista Iberoamericana

de las Ciencias Sociales y

Humanísticas

ISSN: $2395-7972$

Hay tipos de acoso escolar y nosotros representamos algunos (9:103).

Acoso escolar es algo que se vive día con día más en las escuelas (9:9).

Podría decir que este tema es de suma importancia porque muchas personas sufren de él (9:85).

Pues yo aprendí que en el acoso escolar hay víctima, acosador y observador, es repetitivo e intencional (9:27).

El acoso escolar es cuando agreden consecutivamente a alguien, ya sea psicológicamente o físicamente (9:53).

En este sentido, los estudiantes del programa de intervención lograron identificar al acoso escolar como un problema.

Es malo, no lo debo de hacer ni permitir que me lo hagan y, pues, no es justo que lo hagan (9:92).

Es necesario mejorar este tipo de comportamientos dado las consecuencias que existen: te lastiman alguna parte de tu cuerpo y te dejan moretes (9:99), acciones que llevan a la víctima a la depresión; por eso, es tan grave porque lo llevan al suicidio o a matar a toda la escuela (9:97).

En sus relatos, por último, se puede distinguir una percepción positiva del taller: "Tuve buena experiencia y, pues, aprendí demasiado sobre el acoso escolar" (9:9). En sus discursos, de hecho, cambió su valoración de la problemática planteada, pues algunos consideraron que "el acoso escolar es algo muy feo, pero por desgracia es 'normal'" (9:73).

\section{Discusión}

El contexto de este estudio es uno de los más conflictivos y peligrosos de México por la presencia del crimen organizado, los actos de violencia y el vandalismo que proliferan en Ciudad Obregón, Sonora. Debido a esto, los integrantes de la sociedad están expuestos a acontecimientos de esa naturaleza, lo cual tiene efectos negativos en los comportamientos y en las actitudes de los jóvenes, ya que crecer en ambientes violentos donde el vandalismo, los robos, los asaltos y otros actos similares son recurrentes favorece la manifestación de conductas agresivas en edades tempranas (Gómez y Gómez-Maqueo, 2013). Explicado esto, los testimonios recogidos en esta investigación se pueden comprender porque la convivencia en los centros educativos en la actualidad se halla inmersa en un contexto más amplio donde proliferan las conductas violentas (Barquero, 2014). 


\section{Revista Iberoamericana \\ de las Ciencias Sociales y Humanísticas}

ISSN: $2395-7972$

Los participantes, de hecho, reconocen que ellos mismos participan en agresiones físicas y verbales, las cuales son concebidas como rituales para "llevarse bien en la escuela". Estos datos concuerdan con otras investigaciones en las que se reportan conductas violentas en los distintos centros educativos (Maschio et al., 2015; Vega y González, 2016).

Los actos de violencia señalados por los participantes de este trabajo incluyen golpes, groserías, críticas, burlas basadas en las características físicas, exclusión social y acoso en redes sociales, lo cual coincide con lo reportado por Enríquez y Garzón (2015).

Las víctimas, por otra parte, se excluyen del grupo sin comunicar las agresiones recibidas por sus compañeros, situación que es documentada por Betancourt, Arreguín, Aguilar y Dorantes (2015). Estos autores sostienen que los estudiantes realizan acoso psicológico debido a que buscan dañar a las víctimas mediante la exclusión y el aislamiento, lo que afecta su interacción con el grupo.

Para los alumnos las actitudes violentas de las personas se vinculan con la educación y los estilos de crianza de los padres, ya que si estos son agresivos con sus hijos, se estimularán comportamientos similares que luego se reproducirán. De hecho, y según la literatura consultada, el bajo autocontrol de los individuos es un factor asociado a las conductas agresoras debido a que las personas no son capaces de reaccionar de una manera efectiva ante determinadas situaciones (Gómez, Muñoz, Benavidez, Luna y Ortiz, 2013).

Asimismo, una de las consecuencia psicológicas más importantes del acoso reportadas por los alumnos tiene que ver con la depresión de las víctimas, información que concuerda con los reportes de Albores, Sauceda, Ruiz y Roque (2011) y Arroyave (2012). Debido a la complejidad del tema de la violencia en la escuela, es necesario promover intervenciones permanentes con un equipo multidisciplinario y con la participación de los docentes, las autoridades escolares y los padres de familia.

Por último, resulta preocupante que este tipo de acciones sean concebidas como "normales" y se institucionalicen como parte constitutiva de la convivencia en el grupo de pares. Por ende, se recomienda realizar actividades que atiendan dichas conductas agresoras, las cuales no se suelen modificar a corto plazo. También se debe trabajar en un ambiente controlado y con un número reducido de participantes para poder dar seguimiento a todos. 
Revista Iberoamericana

de las Ciencias Sociales y

Humanísticas

ISSN: $2395-7972$

\section{Conclusiones}

El trabajo descrito en las páginas anteriores intentó problematizar los comportamientos agresivos en la escuela, lo cual sirvió para identificar sus manifestaciones, así como los significados que les otorgaban los alumnos. En concreto, los relatos del estudio muestran las formas en que los estudiantes estructuran su dinámica de convivencia con sus pares, lo que se logró explorando sus experiencias en la vida estudiantil cotidiana.

Los participantes señalan que en la convivencia diaria son habituales los grupos conflictivos, así como las dinámicas basadas en juegos bruscos por parte de los involucrados. Asimismo, indican que el contexto escolar se volvía violento debido a las agresiones que forman parte de sus interacciones y a las maneras en que los adolescentes se socializan.

En cuanto a la dinámica de los involucrados, esta se materializa a través de actos violentos, como golpes, groserías, críticas, burlas basadas en las características físicas de los semejantes, exclusión social y acoso en redes sociales.

Los participantes, por otra parte, sostienen que la vida escolar se caracteriza por una convivencia negativa; esto coincide con lo referido en otras investigaciones, las cuales apuntan que las conductas de esa índole suelen formar parte de la cotidianidad de los centros educativos. Asimismo, mencionan que estas actitudes se realizan mediante grupos conflictivos, los cuales hacen que las víctimas se aíslen de los demás y no comuniquen sus vivencias.

Igualmente, llama la atención que algunos alumnos expresan su desaprobación por el comportamiento de sus compañeros, pues no están de acuerdo con la manera violenta como conviven sus pares. Sin embargo, también se destaca que para otros las agresiones no solo son percibidas como hechos "normales", sino que incluso suelen participar en ellas.

En cuanto a la identificación de los actores, los participantes sostienen que los distintos roles presentes en el acoso escolar comparten algunas similitudes. El agresor y el observador, por ejemplo, tienen en común el vigilar a las víctimas y la participación en las agresiones tanto físicas como psicológicas. Por otro lado, tanto la víctima como el observador comparten la idea se sentir miedo a ser agredidos, por lo que suelen ocultar el problema. Sobre las consecuencias psicológicas del acoso escolar, los participantes son conscientes de la depresión que ello puede ocasionar.

En síntesis, al finalizar las sesiones los adolescentes fueron capaces de percibir al acoso escolar como un problema que está presente en la convivencia entre sus compañeros, el cual se manifiesta en agresiones tanto físicas como verbales. Con este trabajo de problematización, 


\section{Revista Iberoamericana}

de las Ciencias Sociales y

Humanísticas

ISSN: $2395-7972$

asimismo, se logró un cambio en la percepción normalizada que tenían los adolescentes sobre los actos de violencia y el acoso en la escuela, situación evidenciada en los relatos de los participantes y en las valoraciones negativas que comentaron al final. Los alumnos, en definitiva, tuvieron una percepción positiva del programa desarrollado, pues comentaron que tuvieron aprendizajes sobre ese tema.

\section{Referencias}

Albores, L., Sauceda, J., Ruiz, S. y Roque, R. (2011). El acoso escolar (bullying) y su asociación con trastornos psiquiátricos en una muestra de escolares en México. Salud Pública de México, 53(3), 220-227.

Alvarado, L. y García, M. (2008). Características más relevantes del paradigma socio-crítico: su aplicación en investigaciones de educación ambiental y de enseñanza de las ciencias realizadas en el doctorado de Educación del Instituto Pedagógico de Caracas. Revista Universitaria de Investigación, 9(2), 187-202.

Arroyave, P. (2012). Factores de vulnerabilidad y riesgo asociados al bullying. Revista CES Psicología, 5(1), 116-125.

Barquero, A. (2014). Convivencia en el contexto familiar: un aprendizaje para construir cultura de paz. Actualidades Investigativas en Educación, 14(1), 1409-4703.

Betancourt, D., Arreguín, P., Aguilar, M. y Dorantes, D. (2015). La sintomatología depresiva y el desempeño académico como factores asociados al bullying en niños. Revista Intercontinental de Psicología y Educación, 17(2), 63-76.

Caballo, V., Calderero, M., Carrillo, G., Salazar, I. e Irurtia, M. (2011). Acoso escolar y ansiedad social en niños (II): una propuesta de intervención en formato lúdico. Psicología Conductual, 19(3), 611-626.

Carrasco, N., García, J. y Zaldívar, F. (2018). Diferencias asociadas a la violencia fillio-parental en función del tipo de familia ("normalizadas" vs "en riesgo") y parentesco de la víctima. Revista de Psicología Clínica con Niños y Adolescentes, 5(3), 30-35.

Cerezo, F. y Sánchez, C. (2013). Eficacia del programa CIP para la mejora de la convivencia escolar y la prevención del bullying en alumnos de educación primaria. Apuntes de Psicología, 31(2), 173-181. 


\section{Revista Iberoamericana}

de las Ciencias Sociales y

Humanísticas

ISSN: $2395-7972$

Córdoba, F., Del Rey, R., Casas, J. y Ortega, R. (2016). Valoración del alumnado de primaria sobre convivencia escolar: el valor de la red de iguales. Psicoperspectivas, Individuo y Sociedad, 15(2), 79-90.

Enríquez, M. y Garzón, F. (2015). El acoso escolar. Saber, Ciencia y Libertad, 10(1), 219-233.

Fernández-Batanero, J. M. (2015). Convivencia escolar. Un estudio en la provincia de Sevilla (España). International Journal of Educational Research and Innovation (IJERI), (3), 7893.

Ferreira, Y. y Reyes, P. (2011). Programa de intervención en habilidades sociales para reducir los niveles de acoso escolar entre pares o bullying. Ajayu Órgano de Difusión Científica del Departamento de Psicología UCBSP, 9(2), 264-283.

Ferrel, F., Cuan, A., Londoño, Z. y Ferrel Ballestas, L. (2015). Factores de riesgo y protectores del bullying escolar en estudiantes con bajo rendimiento de cinco instituciones educativas de Santa Marta, Colombia. Psicogente, 18(33), 188-205. Doi:10.17081/psico.18.33.65

Fierro, M. (2016). Fortalecer la convivencia: (mucho) más que una estrategia remedial frente a la violencia. En Vera, J. y Valdés, A. (eds.), La violencia escolar en México. Temas y perspectivas de abordaje (pp. 213-233). México: CLAVE editorial.

Garaigordobil, M. (2010). Evaluación de los efectos de un programa de prevención de la violencia en factores cognitivos y conductuales desde la percepción subjetiva de los profesores y adolescentes. Anuario de Psicología Clínica de la Salud, 6, 35-43.

Garaigordobil, M. y Maganto, C. (2011). Evaluación de un programa de Educación para la Paz durante la adolescencia: efectos en el autoconcepto y en el concepto de los inmigrantes, de la paz y la violencia. Revista de Investigación en Educación, 9(1), 102-111.

Gibbs, G. (2012). El análisis de datos cualitativos en investigación cualitativa. España: Morata.

Gómez, H. y Gómez-Maqueo, E. (2013). Exposición a la violencia en la comunidad de adolescentes estudiantes: una aproximación cualitativa. Revista Mexicana de Orientación Educativa, 10(25), 68-75.

Gómez, Y., Muñoz, D., Benavidez, J., Luna, E. y Ortiz, L. (2013). Conducta de elección bajo paradigma de autocontrol y desempeño académico en una situación grupal. Revista CES Psicología, 6(2), 105-116.

Güemes, I. (2011). Evaluación de la eficacia del programa "CONVES" para la mejora de la convivencia en educación primaria. Escuela Abierta: Revista de Investigación Educativa, (14), 33-46. 


\section{Revista Iberoamericana}

de las Ciencias Sociales y

Humanísticas

ISSN: 2395 - 7972

Hernández, R. (2015). La positividad del poder: la normalización y la norma. Teoría y Crítica de la Psicología, (3), 81-102.

Instituto Nacional de Estadística y Geografía [Inegi] (2017). Encuesta Nacional de Victimización y Percepción sobre Seguridad Pública (Envipe). Boletín de Prensa. Recuperado de http://www.beta.inegi.org.mx/contenidos/proyectos/enchogares/regulares/envipe/2017/do c/envipe2017_mex.pdf.

Maschio, B., Guimarães, A., Massao, C., Harumi, I., Gramazio, L. y Bezerra, E. (2015). Bullying entre los estudiantes de la educación pública: estudio en una ciudad del norte del Paraná, Brasil. Revista Salusvita, 34(2), 191-203.

Mayora, F. y Castillo, M. (2014). Teoría de la violencia escolar: visión de los actores de una escuela venezolana. Revista Educare, 18(2), 52-72.

Olweus, D. and Limber S. (2017). Some problems with cyberbullying research. Current Opinion in Psychology, 19, 139-143. Doi: 10.1016/j.copsyc.2017.04.012

Orue, I. y Calvete, E. (2012). La justificación de la violencia como mediador de la relación entre la exposición a la violencia y la conducta agresiva en infancia. Psicothema, 24(1), 42-47.

Ossa, C., Figueroa, I. y Rodríguez, F. (2016). La metacognición institucional como herramienta para la mejora de la gestión de la convivencia escolar. Actualidades Investigativas en Educación, 16(3), 1409-4703.

Paulín, H. (2015). “Ganarse el respeto”: un análisis de los conflictos de la sociabilidad juvenil en la escuela secundaria. Revista Mexicana de Investigación Educativa, 20(67), 1105-1130.

Thomas, D. (2003). A General Inductive Approach for Qualitative Data Analises. Nueva Zelanda: University of Auckland. Retrieved from http://www. health.auckland.ac.nz/hrmas/resources/qualdatanalysis.html\#Purposes.

Valdés, A. y Carlos, E. (2014). Relación entre el autoconcepto social, el clima familiar y el clima escolar con el bullying en estudiantes de secundaria. Avances en Psicología Latinoamericana, 32(3), 447-457.

Valdés, A., Bautista, G., Vera, J. y Herrera, J. (2013). Variables que diferencian a estudiantes de secundarias con y sin reportes de bullying en la escuela. Psicología Iberoamericana, 21(1), 32-41.

Valdés, A., Carlos, E. y Torres, G. (2012). Diferencias en la situación socioeconómica, clima y ajuste familiar de estudiantes con reportes de bullying y sin ellos. Psicología desde el Caribe, 29(3), 616-631. 
Vega, M. y González, G. (2016). Bullying en la escuela secundaria. Revista Mexicana de Investigación Educativa, 21(71), 1165-1189.

Viguer, P. y Solé, N. (2015). La participación de las familias en el análisis y la transformación de su realidad mediante un debate familiar sobre valores y convivencia. Universitas Psychologica, 14(1), 355-366.

Wong, D., Cheng, C., Ngan, R. and Stephen, K. (2011). Program Effectiveness of a Restorative Whole-School Approach for Tackling School Bullying in Hong Kong. International Journal of Offender Therapy and Comparative Criminology, 55(6), 846-862. 
Revista Iberoamericana

de las Ciencias Sociales y

Humanísticas

ISSN: 2395 - 7972

\begin{tabular}{|c|c|}
\hline Rol de Contribución & Autor (es) \\
\hline Conceptualización & María de Guadalupe Jocobi Zúñiga \\
\hline Metodología & $\begin{array}{l}\text { María de Guadalupe Jocobi Zúñiga (igual), Oscar Cruz Pérez } \\
\text { (igual) }\end{array}$ \\
\hline Software & NO APLICA \\
\hline Validación & $\begin{array}{l}\text { María de Guadalupe Jocobi Zúñiga (igual), Oscar Cruz Pérez } \\
\text { (igual) } \\
\text { Jesús Ocaña Zúñiga (apoyo) }\end{array}$ \\
\hline Análisis Formal & $\begin{array}{l}\text { María de Guadalupe Jocobi Zúñiga (Principal), Oscar Cruz } \\
\text { Pérez (apoyo), Jesús Ocaña Zúñiga (apoyo) } \\
\text { Germán Alejandro García Lara (apoyo) }\end{array}$ \\
\hline Investigación & $\begin{array}{l}\text { María de Guadalupe Jocobi Zúñiga (Principal), Oscar Cruz } \\
\text { Pérez (Apoyo) }\end{array}$ \\
\hline Recursos & $\begin{array}{l}\text { María de Guadalupe Jocobi Zúñiga, Oscar Cruz Pérez (apoyo) } \\
\text { Jesús Ocaña Zúñiga (apoyo), Germán Alejandro García } \\
\text { Lara(apoyo) }\end{array}$ \\
\hline Curación de datos & $\begin{array}{l}\text { María de Guadalupe Jocobi Zúñiga (Igual), Oscar Cruz Pérez } \\
\text { (Igual) } \\
\text { Jesús Ocaña Zúñiga (Apoyo) }\end{array}$ \\
\hline $\begin{array}{l}\text { Escritura - Preparación del } \\
\text { borrador original }\end{array}$ & $\begin{array}{l}\text { María de Guadalupe Jocobi Zúñiga (principal), Oscar Cruz } \\
\text { Pérez (apoyo) }\end{array}$ \\
\hline $\begin{array}{l}\text { Escritura - Revisión y } \\
\text { edición }\end{array}$ & $\begin{array}{l}\text { María de Guadalupe Jocobi Zúñiga (igual), Oscar Cruz Pérez } \\
\text { (igual) } \\
\text { Jesús Ocaña Zúñiga (apoyo), Germán Alejandro García Lara. } \\
\text { (apoyo) }\end{array}$ \\
\hline Visualización & $\begin{array}{l}\text { María de Guadalupe Jocobi Zúñiga (principal), Oscar Cruz } \\
\text { Pérez (apoyo), Jesús Ocaña Zúñiga (apoyo) } \\
\text { Germán Alejandro García Lara (apoyo) }\end{array}$ \\
\hline Supervisión & $\begin{array}{l}\text { María de Guadalupe Jocobi Zúñiga (igual), Oscar Cruz Pérez } \\
\text { (igual) }\end{array}$ \\
\hline $\begin{array}{l}\text { Administración de } \\
\text { Proyectos }\end{array}$ & María de Guadalupe Jocobi Zúñiga \\
\hline Adquisición de fondos & Oscar Cruz Pérez \\
\hline
\end{tabular}

